\section{Acoso laboral y salud mental en trabajadores chilenos: el papel del género}

\author{
Workplace bullying and mental health in Chilean \\ workers: the role of gender
}

\section{Assédio moral no trabalho e saúde mental em trabalhadores chilenos: o papel do gênero}

Magdalena Ahumada 1

Elisa Ansoleaga ${ }^{2}$

Alvaro Castillo-Carniglia 3,4

\title{
Resumen
}

El acoso laboral ha adquirido relevancia en las últimas décadas, debido a su creciente magnitud y consecuencias negativas en la salud de los trabajadores, existiendo diversos enfoques para estudiarlo. Sin embargo, hasta la fecha en América Latina la evidencia es escasa y focalizada en muestras específicas. El objetivo de este estudio es describir el acoso laboral y su asociación con la salud mental en población asalariada y determinar en qué medida la asociación se modifica según el género. Se realizó una encuesta a una muestra de 1.995 trabajadores y trabajadoras asalariadas de las tres principales áreas metropolitanas de Chile (Gran Santiago, Gran Valparaíso y Gran Concepción) seleccionados al azar en tres etapas (manzanas, viviendas e individuos). La prevalencia de sintomas depresivos, consumo de psicotrópicos y distrés fue de $10,9 \%, 12,8 \%$ y 13\% respectivamente en la muestra total, y existe una fuerte asociación entre variables de salud mental y acoso laboral, aún en los modelos ajustados. Al comparar esta asociación en los modelos estratificados por género, no se observaron diferencias significativas entre hombres y mujeres.

Violencia Laboral; Salud Mental; Salud Ocupacional

Correspondencia

M. Ahumada

Instituto de Salud Pública.

Avenida Marathon 1000, Santiago / Chile.

map.ahumadam@gmail.com

1 Instituto de Salud Pública, Santiago, Chile.

2 Universidad Diego Portales, Santiago, Chile.

${ }^{3}$ Centro de Investigación en Sociedad y Salud, Universidad

Mayor, Santiago, Chile.

${ }_{4}^{4}$ Escuela de Salud Pública, Universidad Mayor, Santiago, Chile. 


\section{Introducción}

El trabajo ocupa un rol central en la vida, por la dedicación temporal, y porque cumple funciones psicosociales como: proporcionar una estructura para la gestión del tiempo, entregar oportunidades para el desarrollo, satisfacción y autoestima, e incluso contribuir a la integración social 1,2. También ha sido identificado como un determinante social de la salud, pudiendo generar efectos positivos o negativos 3,4. En relación con los negativos, la prevalencia de patologías mentales a causa del trabajo ha aumentado a nivel global, lo que se evidencia a través de la cantidad de días perdidos por motivos laborales, aumento de diagnósticos de salud mental 5 y el alto costo que implica para las personas, instituciones y estados. En Europa, superarían los 617.000 millones de euros anuales 6,7, mientras que en Australia, Canadá y Estados Unidos fluctúan entre 8.000 y 300.000 millones de dólares anuales 6 . En Chile, si bien no se han estudiado los costos asociados a patologías mentales, ocupan el primer lugar en gasto por licencias médicas, siendo la población en edad laboral la que presenta mayor prevalencia de sintomatología depresiva 8 .

Para entender la relación entre trabajo y salud mental, se han identificado mecanismos sostenidos de exposición a dimensiones psicosociales del trabajo que generan estrés, dañando la salud mental 9,10,11,12,13. Entre estas dimensiones, Karasek et al. 14 y Siegrist et al. 15 han demostrado resultados consistentes entre la exposición a dimensiones psicosociales y efectos adversos en salud mental: mientras el primero evalúa de qué manera aspectos relacionados con la latitud decisional, demandas psicológicas y apoyo social afectan en los niveles de distrés 16,17; el segundo plantea cómo el desequilibrio entre esfuerzos desplegados y recompensas recibidas puede afectar negativamente la salud física y mental de los trabajadores 18,19,20.

Entre los factores que dañan la salud de los trabajadores, la violencia laboral, también llamada bullying laboral, ha adquirido mayor relevancia en las últimas décadas por su creciente magnitud y consecuencias negativas 21. La Organización Internacional del Trabajo (OIT) lo define como "cualquier acción, todo incidente o comportamiento que no pueda considerarse una actitud razonable y con el cual se ataca, perjudica, degrada o hiere a una persona dentro del marco de su trabajo o debido directamente al mismo" 22. Puede catalogarse por los actos: física, psicológica o verbal, sexual o incivismo, es decir, manifestaciones más sutiles, pero que de igual forma violan las normas sociales 23 . También puede clasificarse por la procedencia de él o la perpetradora, es decir, aquella que se manifiesta internamente en el trabajo y aquella que proviene del exterior (p.ej., desde clientes o usuarios) 22.

De acuerdo con la evidencia internacional, la prevalencia de bullying laboral en Europa fluctúa entre el $14 \%$ y $20 \%$, siendo más reportadas las agresiones verbales y psicológicas 24 . Asimismo, en Estados Unidos y Canadá la prevalencia de agresiones psicológicas y verbales varía entre el 13\% y el $41,4 \% 25,26$. Además, se ha identificado que el bullying laboral es un importante factor de riesgo para la salud de las y los trabajadores, ya que estaría relacionado con el aumento o aparición de patologías mentales y psicosomáticas 27,28 , como el síndrome de estrés post traumático 29,30 , sintomatología depresiva o depresión, aumento de los niveles de distrés 31,32 o uso de psicotrópicos 33 .

En Latinoamérica, los estudios sobre bullying laboral son recientes por lo que la evidencia es escasa y mayoritariamente descriptiva, dificultando la posibilidad de establecer evidencia contundente que permita visibilizar las relaciones entre bullying laboral y salud mental. En una revisión sistemática, Ansoleaga et al. 34 encontraron que existe un predominio de violencia psicológica sobre otros tipos en población laboral latinoamericana, concentrándose en ciertos sectores de la economía (p.ej., sector servicios); las autoras encontraron que los principales efectos sobre la salud mental son: insomnio, irritabilidad y depresión. Asimismo, investigaciones recientes de Brasil, Ecuador y Uruguay han estudiado este fenómeno en distintas muestras de personal sanitario y educacional, encontrando altos niveles de violencia psicológica 35,36, agresión verbal 37 y acoso laboral 38 . En Chile, a pesar de que existen estudios exploratorios, la evidencia principal -previa a este estudio- proviene de una encuesta sobre riesgos psicosociales, calidad del empleo y salud mental con perspectiva de género, cuyos resultados revelaron una importante prevalencia de bullying laboral y diferencias significativas de exposición según el sexo 35 ; y un estudio cualitativo que abordó las manifestaciones de la violencia en tres sectores económicos 39 .

Para entender las manifestaciones de bullying laboral y sus efectos en la salud mental, se han propuesto diversos enfoques. El primero, centrado en la personalidad de la víctima y victimario; el 
segundo, centrado en características inherentes a las interacciones humanas y; el tercero, señala que los factores explicativos se desprenden de la organización del trabajo y cultura organizacional 40. A este último se ha incorporado la presencia de factores sociolaborales, por ejemplo, flexibilización de las condiciones laborales y pobreza, asociados a trabajos informales y precarios 41,42,43. También se considera que esta flexibilización no sólo genera condiciones precarias para una parte importante de la población trabajadora, sino que también reproduce la división sexual del trabajo, derivando en una mayor vulnerabilidad de las mujeres al bullying laboral 44. De esta manera, se ha identificado que mujeres y trabajadores pertenecientes a grupos de menor estatus socioeconómico o a minorías son más vulnerables y se encuentran más expuestas a situaciones de bullying laboral 23,45,46. En el caso de las mujeres, pertenecer a organizaciones dominadas por hombres, ocupar posiciones de menor poder dentro de las instituciones o pertenecer a un grupo minoritario, redundaría en una mayor exposición y en una menor capacidad para defenderse y afrontar la violencia 23,45,46,47,48,49.

El objetivo del artículo es describir la situación de bullying laboral y salud mental de la población asalariada de tres principales áreas metropolitanas de Chile, estimar las asociaciones entre exposición a bullying laboral y salud mental, y determinar en qué medida el efecto de la asociación se modifica según el género. Las hipótesis de este estudio son: (a) la exposición a bullying laboral se asocia con una mayor prevalencia de sintomatología depresiva, consumo de psicotrópicos y distrés psicológico en trabajadores asalariados de zonas urbanas; y (b) la asociación entre bullying laboral y salud mental es más fuerte en mujeres que en hombres.

Se busca contribuir a la comprensión del fenómeno, permitiendo visibilizar la exposición de bullying laboral y sus efectos en la salud mental en una muestra multiestamental, en las tres regiones de Chile con mayor población trabajadora. Lo anterior es relevante, ya que hasta la fecha existe escasa evidencia empírica en países en vías de desarrollo, y el reconocimiento de este problema y su afrontamiento a través de políticas públicas es un asunto reciente.

\section{Materiales y método}

En el marco del proyecto FONDECYT Regular no 1170239, se realizó un estudio transversal en una muestra multiestamental y aleatoria de trabajadores y trabajadoras asalariadas de las tres principales áreas metropolitanas de Chile (Gran Santiago, Gran Valparaíso y Gran Concepción). El tamaño muestral estimado fue de 2.000 casos, y consideró un error absoluto del 3\% para una proporción del $50 \%$ (varianza máxima), un efecto de diseño de 1,2 y un nivel de significancia de 0,05. La selección de la muestra fue de tipo probabilística y estratificada en tres etapas de selección aleatoria (manzana, vivienda e individuo). En cada manzana se seleccionaron seis viviendas con reemplazo, y dentro de cada vivienda a un individuo mediante tabla de Kish. Este método de selección de participantes y su asegurada aleatoriedad permitieron evitar el sesgo de selección. Dada la menor proporción de mujeres en el mercado laboral formal en Chile, las trabajadoras mujeres fueron sobrerrepresentadas para obtener una proporción equivalente por sexo. Para esto se estableció en el protocolo de terreno que, en caso de no haber encuestado al menos una mujer en las primeras tres viviendas de cada manzana, se debía seleccionar a una trabajadora mujer en la 4ta y 5 ta vivienda visitada. Los casos fueron ponderados en base a los parámetros de la Encuesta Nacional de Empleo de Chile, actualizada al trimestre enero-marzo del año 2018, con relación a la proporción ocupacional según sexo y región 50.

Los criterios de elegibilidad fueron hombres y mujeres asalariados o dependientes, de 20 años o más, residentes de una de las tres principales áreas metropolitanas de Chile. Se excluyó a trabajadores independientes, personal de las fuerzas armadas y de orden y servicio doméstico.

La encuesta consideró una entrevista cara a cara, utilizando método CAPI (computer-assisted personal interview) en las viviendas de los participantes. Los/as encuestadores/as fueron capacitados/as por el equipo de investigación acerca de la temática y tipo de preguntas a realizar. El periodo de captación se realizó entre marzo y mayo del 2018, obteniendo 1.995 casos válidos y una tasa de respuesta de $87,3 \%$.

El protocolo de este estudio fue revisado y aprobado por el Comité de Ética de Investigación de la Universidad Diego Portales. 


\section{Instrumentos y variables}

Para el análisis se consideraron tres desenlaces: (a) uso de psicotrópicos, medido a través de tres preguntas que remiten al consumo de ansiolíticos, hipnóticos y antidepresivos en el último año. Se consideró consumo el indicar al menos un tipo; (b) distrés psicológico, medido a través de la escala K6, validada en Chile por Ansoleaga et al. ${ }^{51}$ y que consta de seis ítems relativos a la presencia e intensidad de síntomas como: sentirse nervioso, cansado o sin esperanzas, en los últimos 30 días 52,53. Se consideró presencia de distrés en aquellos casos que presentaban siete o más puntos en la escala, cuyo rango fue de 0 a 24. La consistencia interna de esta escala en la muestra del presente estudio de $\alpha=0,85$; y (c) síntomas depresivos, evaluados en base a la presencia de al menos uno de dos criterios diagnósticos incluidos en el Manual Diagnóstico y Estadístico de los Trastornos Mentales (DSM-IV), es decir, sentirse triste o deprimido por dos semanas consecutivas o más, y sentir pérdida de interés por la mayoría de las cosas que les gustaba hacer por un periodo de dos semanas seguidas o más. Estas preguntas han sido utilizadas en otros estudios chilenos para evaluar sintomatología depresiva 54,55 .

La exposición (acoso laboral) fue evaluada a través del cuestionario NAQ-R (Negative Acts Questionnaire-Revised), compuesto por 22 ítems que miden la frecuencia de exposición a conductas negativas en los últimos seis meses mediante una escala tipo Likert ("nunca" a "diariamente"). Los ítems están formulados en términos comportamentales y no mencionan la palabra acoso ni similares. La versión angloamericana fue traducida al español por González \& Graña 56, y validada en Chile por GonzálezSanta Cruz 57 . Se consideraron como expuestos a bullying laboral a aquellos que presentaran al menos dos conductas con una respuesta igual o mayor a "al menos 1 vez a la semana" 58. La consistencia interna de esta escala fue de $\alpha=0,93$.

Para incorporar la dimensión género, se consideró la variable sexo, como potencial modificador de efecto, razón por la cual se estratificaron los análisis entre hombres y mujeres y se compararon los resultados.

En los modelos ajustados se consideraron como confusores potenciales: ocupación, tipo de contrato, tipo de jornada laboral y carga doméstica.

Ocupación fue creada por medio de la pregunta acerca de la ocupación del encuestado, se categorizó a aquellos trabajadores en empleos de baja cualificación (operarios, no cualificados, y conductores de transporte) como "no cualificados" (1) y como "cualificados" (0) a trabajadores en empleos de alta cualificación (empleados de oficina, de servicios, vendedores, jefaturas intermedias y profesionales).

La variable tipo de contrato a través de la pregunta "¿Qué tipo de contrato o acuerdo de trabajo tiene?", cuyas respuestas son: "Contrato indefinido" (1), “Con plazo de término (temporada, obra o faena)" (2), "A contrata” (3), “Contrato a honorarios (boletea)”(4) y "Otro”(5). Para este estudio las respuestas se dicotomizaron en contratos indefinidos (0) y contratos con plazo fijo u otros (1).

La variable tipo de jornada laboral deriva de la pregunta sobre el horario de trabajo, donde se categorizó la jornada diurna (1) y el resto de las jornadas distintas a esta (2), es decir, turnos nocturnos, rotativos, medio tiempo y variable.

La carga doméstica fue creada por medio de la pregunta "Y durante la semana, ¿usted dedica tiempo para hacer tareas domésticas y/o de cuidado, pero no remunerado?”, donde las respuestas afirmativas se codificaron como presencia del atributo (1) y las negativas como ausencia (0).

\section{Análisis}

En primer lugar, se realizó un análisis univariante de las variables en estudio (NAQ-R, consumo de psicotrópicos, síntomas depresivos y distrés psicológico) y de las variables de ajuste a considerar en los modelos estadísticos (ocupación, tipo de contrato, horario de trabajo y carga doméstica).

Se desarrolló un modelo bivariado entre bullying laboral y variables vinculadas de salud mental (consumo de psicotrópicos, síntomas depresivos y distrés psicológico), a través de modelos de regresión log-binomial estratificados por sexo. Posteriormente, se realizaron análisis multivariados, incluyendo variables que podrían confundir la relación entre bullying laboral y salud mental, y que son identificadas como relevantes, según la literatura científica en esta área (ocupación, tipo contrato, carga doméstica y jornada). Las medidas de asociación reportadas en los análisis bi y multivariados fueron razones de prevalencia (RP) crudas y ajustadas y sus respectivos intervalos de $95 \%$ de confianza 
(IC95\%). La varianza de todos los modelos se estimó mediante serie linealizada de Taylor, que considera las distintas etapas del diseño muestral.

La comparación entre los coeficientes de los modelos para hombres y mujeres se realizó mediante la prueba de Wald, cuya hipótesis nula es que los coeficientes de los modelos son iguales. Todos los análisis se realizaron con el paquete estadístico Stata versión 15 (https://www.stata.com).

\section{Resultados}

Del total de mujeres, un 17,8\% no completó la educación media, mientras que del total de los hombres, un $24 \%$ está en la misma situación. En cuanto a la ocupación, un $41 \%$ del total de mujeres son trabajadoras no cualificadas y un $12 \%$ tiene una jornada distinta a la diurna. En cuanto al total de hombres, la mitad tiene una ocupación no cualificada, y un $23 \%$ tiene una jornada distinta a la jornada regular (Tabla 1).

Parte importante de la muestra $(28,8 \%)$ tiene contrato a plazo fijo, por obra o faena, es decir, distinto a un contrato indefinido. En cuanto a la "carga doméstica", la gran mayoría de las mujeres (89,4\% del total) tiene carga doméstica, además de la carga laboral. En cambio, del total de hombres, un $67,6 \%$ se encuentra en la misma situación.

En relación con los indicadores de salud mental, la supera el 10\% en el total de la muestra. Esta prevalencia cambia al observar diferenciadamente los sexos, en tanto un $15,8 \%, 18,3 \%$ y $18,2 \%$ del total de mujeres presenta síntomas depresivos, consume psicotrópicos y experimenta distrés psicológico, respectivamente. En el caso de los hombres, del total un 7,6\%, 9\% y 9,6\% tiene síntomas depresivos, consume psicotrópicos y experimenta distrés psicológico, respectivamente.

Por último, del total de la muestra un 10,2\% de los trabajadores está expuesto a bullying laboral. Sin embargo, del total de mujeres un 9,8 está expuesta, y del total de hombres, un 10,5\% está expuesto a bullying laboral.

En el modelo crudo (Tabla 2) se puede observar que, en el total de la muestra, la exposición a bullying laboral aumenta entre 2 y 3 veces el riesgo de presentar distrés psicológico $(\mathrm{RP}=2,95$; IC95\%: 2,23-3,90), consumir psicotrópicos ( $\mathrm{RP}=2,75$; IC95\%: 2,06-3,66) y presentar síntomas depresivos $(\mathrm{RP}=3,08$; IC95\%: 2,26-4,19) con respecto a trabajadores y trabajadoras no expuestos.

Tabla 1

Descripción de la muestra.

\begin{tabular}{lcccccc}
\hline & \multicolumn{2}{c}{ Hombres } & \multicolumn{2}{c}{ Mujeres } & \multicolumn{2}{c}{ Total } \\
& $\mathbf{n}$ & $\%$ & $\mathbf{n}$ & $\%$ & $\mathbf{n}$ & $\%$ \\
\hline Zona & & & & & & \\
$\quad$ Gran Santiago & 414 & 64,4 & 385 & 66,0 & 799 & 65,0 \\
$\quad$ Gran Valparaíso & 295 & 16,8 & 302 & 16,8 & 597 & 16,8 \\
$\quad$ Gran Concepción & 262 & 18,8 & 337 & 17,2 & 599 & 18,2 \\
Educación (menos que educación secundaria) & 231 & 24,0 & 172 & 17,8 & 403 & 21,5 \\
Ocupación (no cualificado) & 561 & 56,3 & 421 & 41,0 & 982 & 50,1 \\
Tipo de contrato (contrato definido) & 298 & 29,6 & 292 & 27,5 & 590 & 28,8 \\
Jornada (turnos rotativos) & 232 & 23,0 & 130 & 12,0 & 362 & 18,6 \\
Carga doméstica & 622 & 67,6 & 902 & 89,4 & 1.524 & 76,4 \\
Síntomas depresivos & 67 & 7,6 & 149 & 15,8 & 216 & 10,9 \\
Uso de psicotrópicos & 86 & 9,0 & 179 & 18,3 & 265 & 12,8 \\
Distrés psicológico & 80 & 9,6 & 166 & 18,2 & 246 & 13,0 \\
Violencia laboral & 98 & 10,5 & 100 & 9,8 & 198 & 10,2 \\
\hline
\end{tabular}

Nota: los porcentajes están ponderados para representar adecuadamente la distribución poblacional por sexo y edad en la población trabajadora de estas tres áreas urbanas. 
Tabla 2

Asociación entre exposición a violencia laboral e indicadores de salud mental en población trabajadora de Chile.

\begin{tabular}{|c|c|c|c|c|c|c|c|}
\hline \multirow[t]{3}{*}{ Indicadores de salud mental } & \multicolumn{7}{|c|}{ Análisis bivariado * } \\
\hline & \multicolumn{2}{|c|}{ Muestra total } & \multicolumn{2}{|c|}{ Hombres } & \multicolumn{2}{|c|}{ Mujeres } & \multirow[t]{2}{*}{ Hombres vs. mujeres } \\
\hline & RP & IC95\% & RP & IC95\% & $\mathbf{R P}$ & IC95\% & \\
\hline \multirow[t]{2}{*}{ Distrés psicológico } & 2,95 & $2,23-3,90$ & 4,00 & $2,58-6,22$ & 2,27 & $1,61-3,20$ & $x^{2}=3,96$ \\
\hline & & & & & & & $p=0,0468$ \\
\hline \multirow[t]{2}{*}{ Uso de psicotrópicos } & 2,75 & $2,06-3,66$ & 2,78 & $1,69-4,57$ & 2,79 & $2,04-3,83$ & $x^{2}=0,00$ \\
\hline & & & & & & & $p=0,9857$ \\
\hline \multirow[t]{2}{*}{ Síntomas depresivos } & 3,08 & $2,26-4,19$ & 3,14 & $1,82-5,42$ & 3,08 & $2,19-4,33$ & $\chi^{2}=0,00$ \\
\hline & & & & & & & $p=0,9512$ \\
\hline
\end{tabular}

IC95\%: intervalo de 95\% de confianza; RP: razón de prevalencia.

* Modelo de regresión log-binomial.

Al estratificar por sexo se encuentra que la fuerza del efecto del bullying laboral en la salud mental se mantiene, sin embargo, existirían diferencias. En el segmento de mujeres, la exposición a bullying laboral aumenta el riesgo entre 2 y 3 veces de presentar síntomas depresivos ( $\mathrm{RP}=3,08$; IC95\%: 2,194,33), consumir psicotrópicos ( $\mathrm{RP}=2,79$; IC95\%: 2,04-3,83) y de presentar distrés ( $\mathrm{RP}=2,27$; IC95\%: 1,61-3,20), en comparación con las trabajadoras que no están expuestas. En el segmento de hombres, los expuestos a bullying laboral tienen 2 y 3 veces más riesgo de presentar síntomas depresivos $(\mathrm{RP}=$ 3,14; IC95\%: 1,82-5,42) y consumir psicotrópicos ( $\mathrm{RP}=2,78$; IC95\%: 1,69-4,57) respectivamente, además de aumentar 4 veces el riesgo de presentar distrés ( $\mathrm{RP}=4,01$; IC95\%: 2,58-6,23).

Al comparar los coeficientes de los modelos para ambos sexos se observa que, sólo en el distrés psicológico, las diferencias son significativas para hombres y mujeres. Tanto para uso de psicotrópicos, como para síntomas depresivos, no hay evidencia de que la asociación con bullying laboral sea más fuerte en mujeres que en hombres.

De acuerdo a lo observado (Tabla 3), la fuerza de la asociación entre bullying laboral y salud mental se mantiene en la muestra total, es decir, aumenta entre 2 y 3 veces el riesgo de presentar distrés psicológico ( $\mathrm{RP}=2,90$; IC95\%: 2,18-3,86), consumir psicotrópicos ( $\mathrm{RP}=2,72$; IC95\%: 2,04-3,62) y presentar síntomas depresivos ( $\mathrm{RP}=3,00$; IC95\%: 2,21-4,10), con respecto a trabajadores y trabajadoras no expuestos, aun ajustando por el resto de las variables del modelo.

La fuerza de la asociación se mantiene al estratificar por sexo. Por un lado, en el segmento de mujeres, la exposición a bullying laboral aumenta el riesgo entre 2 y 3 veces el riesgo de presentar síntomas depresivos ( $\mathrm{RP}=3,15$; IC95\%: 2,24-4,43), consumir psicotrópicos ( $\mathrm{RP}=2,81$; IC95\%: 2,06-3,84) y presentar distrés ( $\mathrm{RP}=2,33$; IC95\%: 1,64-3,30), en comparación con las trabajadoras que no están expuestas a bullying laboral. Por otro lado, en el segmento de hombres, los expuestos a bullying laboral también tienen más riesgo de presentar síntomas depresivos ( $\mathrm{RP}=2,97$; IC95\%: 1,75-5,10), consumir psicotrópicos ( $\mathrm{RP}=2,77$; IC95\%: 1,68-4,59) y de presentar distrés (RP = 3,90; IC95\%: 2,46-6,20).

Al comparar los coeficientes de los modelos para ambos sexos, se observa que no hay evidencia de que la asociación entre bullying laboral y las variables de salud mental consideradas sean más fuertes en mujeres que en hombres. 
Tabla 3

Asociación entre exposición a violencia laboral e indicadores de salud mental en población trabajadora de Chile, ajustado por variables de interés.

\begin{tabular}{|c|c|c|c|c|c|c|c|}
\hline \multirow[t]{3}{*}{ Indicadores de salud mental } & \multicolumn{7}{|c|}{ Análisis multivariado *,** } \\
\hline & \multicolumn{2}{|c|}{ Muestra total } & \multicolumn{2}{|c|}{ Hombres } & \multicolumn{2}{|c|}{ Mujeres } & \multirow[t]{2}{*}{ Hombres vs. mujeres } \\
\hline & $\mathbf{R P}$ & IC95\% & $\mathbf{R P}$ & IC95\% & RP & IC95\% & \\
\hline \multirow[t]{2}{*}{ Distrés psicológico } & 2,90 & $2,18-3,86$ & 3,90 & $2,46-6,20$ & 2,33 & $1,64-3,30$ & $\chi^{2}=3,06$ \\
\hline & & & & & & & $p=0,0802$ \\
\hline \multirow[t]{2}{*}{ Uso de psicotrópicos } & 2,72 & $2,04-3,62$ & 2,77 & $1,68-4,59$ & 2,81 & $2,06-3,84$ & $\chi^{2}=0,00$ \\
\hline & & & & & & & $p=0,9655$ \\
\hline \multirow[t]{2}{*}{ Síntomas depresivos } & 3,00 & $2,21-4,10$ & 2,97 & $1,75-5,10$ & 3,15 & $2,24-4,43$ & $\chi^{2}=0,03$ \\
\hline & & & & & & & $p=0,8623$ \\
\hline
\end{tabular}

IC95\%: intervalo de 95\% de confianza; RP: razón de prevalencia.

* Modelo de regresión log-binomial;

** Modelo ajustado por "ocupación", "tipo de contrato", "jornada” y “carga doméstica".

\section{Discusión}

La prevalencia de síntomas depresivos, consumo de psicotrópicos y distrés es superior a 10\% en la muestra total, similar a resultados internacionales. Por ejemplo, según la Organización Mundial de la Salud (OMS), la prevalencia de trastornos depresivos en otras regiones fluctúa entre 9\% y $27 \% 55$. Otros estudios indican que el consumo de psicotrópicos sería mayor a 11\%, siendo las mujeres las que más consumen 59 , y la prevalencia de distrés estaría entre $5 \%$ y $27 \%$, existiendo diferencias en los modos de medición 60 .

Puede establecerse una asociación entre exposición a bullying laboral y salud mental, ya que en ambos sexos el estar expuestos a bullying laboral aumenta entre 2 y 3 veces el riesgo de presentar problemas de salud mental. Además, al ajustar por variables vinculadas a desigualdades ocupacionales, el tamaño del efecto de la asociación se mantiene, lo que indica que estas covariables no logran confundir el efecto del bullying laboral en la salud mental, confirmando lo planteado en la evidencia, en relación a los daños que puede causar el bullying laboral en la salud mental 61,62, y que incluso se considera uno de los problemas más devastadores en comparación con otras manifestaciones del estrés laboral 60 . En definitiva, los resultados obtenidos son concordantes con lo que muestra la evidencia, en tanto, la exposición a bullying laboral aumentaría la posibilidad de presentar síntomas depresivos, distrés psicológico y/o de usar psicotrópicos 27,28,29,30,31,32,33.

Al comparar los modelos de hombres y mujeres el género no cumpliría un rol modificador. Si bien estos resultados no concuerdan con evidencia latinoamericana, en donde sí se han encontrado diferencias de género 35,36 , se debe considerar que corresponden a muestras específicas y no directamente comparables. Resultados similares a los de este estudio han sido observados en estudios internacionales. Feijó et al. 63 revisaron 135 estudios sobre bullying laboral y encontraron que si bien, en la mayoría de los estudios, la exposición a violencia en mujeres es mayor, en 11 artículos no se evidenció asociación entre género y bullying laboral.

Se debe considerar que las mujeres a menudo se estudian como si fueran una sola categoría, pese a que, en estudios de salud ocupacional con perspectiva de género, se plantea que sería una categoría fragmentada con múltiples aristas relevantes para la investigación y que incluso dentro del trabajo, tendrían importantes diferencias 64. Sumado a esto, las dinámicas de género podrían afectar a ambos sexos, en especial en sectores donde hay una clara dominación de un género sobre el otro. Por ejemplo, se ha encontrado que las mujeres sufren más bullying laboral en sectores vinculados a la tecnología, así como los hombres tendrían alta prevalencia en educación y salud 65 .

Otra explicación se refiere a la posibilidad de que las mujeres normalicen el bullying laboral, especialmente en sectores donde predominan como población trabajadora. Existiría una naturalización 
de la subordinación, a través de la reproducción de patrones de desigualdad social, que harían parecer discriminaciones sutiles como inexistentes o como parte del trabajo que ejercen 66. Esto releva la necesidad de realizar análisis más específicos, como diferenciar por sectores económicos u ocupaciones, para visibilizar desigualdades sociolaborales que se encuentran enraizadas en el complejo sistema de relaciones de género de la sociedad 67,68.

Estos resultados contribuyen a visibilizar la relación entre bullying laboral y salud mental en Chile, y cómo otras dimensiones pueden modificar su asociación, sin reducir la complejidad que tiene este fenómeno. Se busca relevar la necesidad de informar, prevenir e intervenir en las organizaciones, a fin de proteger la salud psicológica de los trabajadores y trabajadoras, considerando las graves consecuencias que trae la exposición a bullying laboral. Además, estos resultados buscan posicionar la temática a nivel de vigilancia de la salud, especialmente en Latinoamérica, en donde este estudio puede brindar luces a los formuladores de políticas públicas y a profesionales que intervienen en el ámbito laboral.

Este estudio debe ser interpretado a la luz de las siguientes limitaciones. Primero, el diseño es de tipo transversal, lo que impide examinar de forma empírica la secuencia temporal entre exposición a bullying laboral y la ocurrencia de problemas de salud mental. Segundo, la clasificación binaria de bullying laboral es una de las miradas utilizadas para revisar la exposición, existiendo otros criterios operacionales, como el uso de curvas ROC, o análisis de clases latentes que suelen asociarse a representaciones más procesuales de los fenómenos 69 . Por último, una limitación, que se asume como una guía para estudios posteriores, es ahondar en otros aspectos de desigualdades sociales y ocupacionales como el trabajo informal, estatus migratorio, barreras lingüísticas, diferencias étnicas, preferencias sexuales, identidades de género, entre otras 70,71. Una caracterización comprehensiva contribuiría a la generalización de resultados, así como a la comprensión de su manifestación en grupos particulares de la sociedad.

\section{Conclusiones}

La exposición a bullying laboral se asocia con una mayor prevalencia de sintomatología depresiva, consumo de psicotrópicos y distrés psicológico en trabajadores asalariados de zonas urbanas. La ausencia de diferencias significativas de género puede deberse a una falta de precisión respecto de sectores ocupacionales específicos en los cuales se observa mayor acoso laboral en mujeres que en hombres. Contrario a lo observado en estudios similares, no se observaron diferencia en estas asociaciones entre hombres y mujeres.

\section{Colaboradores}

Todos los autores contribuyeron con el diseño, análisis, redacción y revisión del documento y aprobaron la versión final.

\section{Informaciones adicionales}

ORCID: Magdalena Ahumada (0000-0003-2838-

0458); Elisa Ansoleaga (0000-0003-0182-0126);

Alvaro Castillo-Carniglia (0000-0002-3016-890X).

\section{Agradecimientos}

Al Fondo Nacional de Desarrollo Científico y Tecnológico (FONDECYT) por el apoyo financiero. 


\section{Referencias}

1. Brito MJM, Vianna PCM, Silva TC, Costa ASSM, Santos FX. O significado do trabalho para profissionais de saúde mental. J Health Sci Inst 2010; $28: 165-8$.

2. Da Rosa S, Chalfin M, Baasch D, Soares J. Sentidos y significados del trabajo: un análisis con base en diferentes perspectivas. Univ Psychol 2011; 10:175-88.

3. Gil Hernández F. Tratado de medicina del trabajo. Amsterdam: Elsevier; 2011.

4. Benach J, Muntaner J. Empleo, trabajo y desigualdades en salud: una visión global. https://www.researchgate.net/publica tion/318361793_Empleo_Trabajo_Y_Des igualdades_en_Salud_Una_vision_Global (accedido el 27/Jun/2019).

5. Organización Internacional del Trabajo. Estrés en el trabajo: un reto colectivo. http://www. ilo.org/safeday (accedido el 28/Jun/2019).

6. Hassard J, Teoh K, Cox T, Dewe P. La estimación del coste del estrés y los riesgos psicosociales relacionados con el trabajo. Bilbao: Agencia Europea para la Seguridad y la Salud en el Trabajo; 2014.

7. Leka S, Jain A, Iavicoli S, Di Tecco C. An evaluation of the policy context on psychosocial risks and mental health in the workplace in the European Union: achievements, challenges, and the future. Biomed Res Int 2015; 2015:213089.

8. Errázuriz P, Valdés C, Vöhringer PA, Calvo E. Financiamiento de la salud mental en Chile: una deuda pendiente. Rev Med Chile 2015; 143:1179-86.

9. LaMontagne AD, Milner A, Krnjacki L, Schlichthorst M, Kavanagh A, Page K, et al. Psychosocial job quality, mental health, and subjective wellbeing: a cross-sectional analysis of the baseline wave of the Australian Longitudinal Study on Male Health. BMC Public Health 2016; 16 Suppl 3:33-41.

10. Milner A, King T, LaMontagne AD, Bentley R, Kavanagh A. Men's work, women's work, and mental health: a longitudinal investigation of the relationship between the gender composition of occupations and mental health. Soc Sci Med 2018; 204:16-22.

11. Reimann M. The moderating role of overcommitment in the relationship between psychological contract breach and employee mental health. J Occup Health 2016; 58:425-33.

12. Cadieux N, Marchand A. Psychological distress in the workforce: a multilevel and longitudinal analysis of the case of regulated occupations in Canada. BMC Public Health 2014; 14:808.

13. Koch AK, Adler M. Emotional exhaustion and innovation in the workplace: a longitudinal study. Ind Health 2018; 56:524-38.

14. Karasek R, Brisson C, Kawakami N, Houtman I, Bongers P, Amick B. The Job Content Questionnaire (JCQ): an instrument for internationally comparative assessments of psychosocial job characteristics. J Occup Health Psychol 1998; 3:322-55
15. Siegrist J, Wege N, Pühlhofer F, Wahrendorf $\mathrm{M}$. A short generic measure of work stress in the era of globalization: effort-reward imbalance. Int Arch Occup Environ Health 2009; 82:1005-13.

16. Chungkham HS, Ingre M, Karasek R, Westerlund $\mathrm{H}$, Theorell T. Factor structure and longitudinal measurement invariance of the demand control support model: an evidence from the Swedish Longitudinal Occupational Survey of Health (SLOSH). PLoS One 2013; 8:e70541.

17. Fransson EI, Nyberg ST, Heikkilä K, Alfredsson L, Bacquer DD, Batty GD, et al. Comparison of alternative versions of the job demandcontrol scales in 17 European cohort studies: the IPD-Work consortium. BMC Public Health 2012; 12:62.

18. Siegrist J. Effort-reward imbalance at work and cardiovascular diseases. Int J Occup Med Environ Health 2010; 23:279-85.

19. Wege N, Li J, Siegrist J. Are there gender differences in associations of effort-reward imbalance at work with self-reported doctordiagnosed depression? Prospective evidence from the German Socio-Economic Panel. Int Arch Occup Environ Health 2018; 91:435-43.

20. Siegrist J, Li J. Associations of extrinsic and intrinsic components of work stress with health: a systematic review of evidence on the effortreward imbalance model. Int J Environ Res Public Health 2016; 13:432.

21. Chapell D, Di Martino V. Violence at work. Geneva: International Labour Organization; 2006.

22. Organización Internacional del Trabajo. La violencia en el trabajo. v. 4. Educación obrera. http://www.ilo.org/wcmsp5/groups/public/@ ed_dialogue/@actrav/documents/publication/ wcms_117581.pdf (accedido el 03/Mar/2020).

23. Cortina LM. Unseen injustice: incivility as modern discrimination in organizations. Acad Manage Rev 2008; 33:55-75.

24. Eurofound. Violence and harassment in an european workplaces: extend, impacts and policies. http://www.eurofound.europa.eu/sites/ default/files/ef_comparative_analytical_re port/field_ef_documents/ef1473en.pdf (accedido el 03/Mar/2020).

25. Kelloway EK, Barling J, Hurrell JJ. Handbook of workplace violence. London: Sage Publications; 2006.

26. Hango D, Moyser M. Harassment in Canadian workplaces. Insights on Canadian Society 2018; (1). https://www150.statcan.gc.ca/n1/ pub/75-006-x/2018001/article/54982-eng. pdf.

27. Nielsen MB, Magerøy N, Gjerstad J, Einarsen S. Workplace bullying and subsequent health problems. Tidsskr Nor Legeforen 2014; 134:1233-8. 
28. Hsieh Y-H, Wang H-H, Ma S-C. The mediating role of self-efficacy in the relationship between workplace bullying, mental health and an intention to leave among nurses in Taiwan. Int J Occup Med Environ Health 2019; 32:245-54.

29. Tatar ZB, Yüksel Ş. Mobbing at workplace: psychological trauma and documentation of psychiatric symptoms. Noro Psikiyatr Ars 2019; 56:57-62.

30. Malinauskiene V, Einarsen S. Workplace bullying and post-traumatic stress symptoms among family physicians in Lithuania: an occupation and region specific approach. Int J Occup Med Environ Health 2014; 27:919-32.

31. Salas ML, Quezada S, Basagoitia A, Fernandez T, Herrera R, Parra M, et al. Working conditions, workplace violence, and psychological sistress in Andean miners: a cross-sectional study across three countries. Ann Glob Health 2015; 81:465-74.

32. Taniguchi T, Takaki J, Hirokawa K, Fujii Y, Harano K. Associations of workplace bullying and harassment with stress reactions: a twoyear follow-up study. Ind Health 2016; 54:1318.

33. Niedhammer I, David S, Degioanni S, Drummond A, Philip P, Acquarone D, et al. Workplace bullying and psychotropic drug use: the mediating role of physical and mental health status. Ann Occup Hyg 2010; 55:152-63.

34. Ansoleaga E, Gómez-Rubio C, Mauro A. Workplace violence in Latin American: a review of cientific evidence. Vertex 2015; 26:444-52.

35. Lara E, Pando M. El mobbing y los síntomas de estrés en docentes universitarios del sector público. Cienc Trab 2014; 16:43-8.

36. Fontes K, Santana R, Pelloso S, Carvalho M. Factores asociados al acoso moral en el ambiente laboral del enfermero. Rev Latinoam Enferm 2013; 21:758-64.

37. Silva I, Aquino E, Pinto I. Violência no trabalho em saúde: a experiência de servidores estaduais da saúde no Estado da Bahia, Brasil. Cad Saúde Pública 2014; 30:2112-22.

38. Buunk AP, Franco S, Dijkstra P, Zurriaga R. Mobbing in schools and hospitals in Uruguay. J Interpers Violence 2017; 32:623-34.

39. Gideon J. Handbook on gender and health. London: Edward Elgar Publishing; 2016.

40. Ansoleaga E, Díaz X, Mauro A, Toro JP. Dimensiones organizacionales de la violencia en el trabajo en Chile considerando diferencias ocupacionales y de género. Estudio en tres sectores económicos. http://cem.cl/wp-content/ uploads/2019/01/Documento-Cientificodovt.pdf.pdf (accedido el 15/Mar/2020).

41. Einarsen S. Harassment and bullying at work. Aggress Violent Behav 2000; 5:379-401.

42. Llosa-Fernández JA, Menéndez-Espina S, Agulló-Tomás E, Rodríguez-Suárez J. Job insecurity and mental health: a meta-analytical review of the consequences of precarious work in clinical disorders. An Psicol 2018; 34:21123.
43. Quinlan M. The effects of non-standard forms of employment on worker health and safety. https://www.ilo.org/travail/whatwedo/publi cations/WCMS_443266/lang--en/index.htm (accedido el 18/May/2019).

44. Millones M. Límites del trabajo decente: la precarización laboral como problema estructural en América Latina. Gaceta Laboral 2012; 18:87-106.

45. Diaz X, Mauro A, Ansoleaga E, Toro JP. Violencia de género en el trabajo en Chile. Un campo de estudio ignorado. Cienc Trab 2017; 19:42-8.

46. García Johnson CP, Otto K. Better together: a model for women and LGBTQ equality in the workplace. Front Psychol 2019; 10:272.

47. Krieger N, Waterman PD, Hartman C, Bates LM, Stoddard AM, Quinn MM, et al. Social hazards on the job: workplace abuse, sexual harassment, and racial discrimination - a study of Black, Latino, and White low-income women and men workers in the United States. Int J Health Serv 2006; 36:51-85.

48. Hoel H, Salin D. Organisational antecedents of workplace bullying. In: Einarsen S, Hoel $\mathrm{H}$, Cooper C, editors. Bullying and emotional abuse in the workplace. London: CRC Press; 2002. p. 221-36.

49. Einarsen S, Hoel H, Zapt D, Cooper CL. Bullying and harassment in the workplace. London: CRC Press; 2011.

50. Instituto Nacional de Estadísticas. Encuesta suplementaria de ingresos 2017. http://www. ine.cl (accedido el 06/Jun/2019).

51. Ansoleaga E, Montaño R, Michel V. Validation of two complementary instruments for measuring work stress in Chilean workers. Scandinavian Journal of Organizational Psychology 2013; 5:5-14.

52. Ansoleaga E, Ahumada M, González-Santa Cruz A. Association of workplace bullying and workplace vulnerability in the psychological distress of Chilean workers. Int J Environ Res Public Health 2019; 16:4039.

53. Kessler RC, Andrews G, Colpe LJ, Hiripi E, Mroczek DK, Normand SLT, et al. Short screening scales to monitor population prevalences and trends in non-specific psychological distress. Psychol Med 2002; 32:959-76.

54. Ansoleaga E, Vézina M, Montaño R. Síntomas depresivos y distrés laboral en trabajadores chilenos: condiciones diferenciales para hombres y mujeres. Cad Saúde Pública 2014; 30:107-18.

55. Ansoleaga E. Indicadores de salud mental asociados a riesgo psicosocial laboral en un hospital público. Rev Med Chile 2015; 143:47-55.

56. González D, Graña JL. El acoso psicológico en el lugar de trabajo: prevalencia y análisis descriptivo de una muestra multiocupacional. Psicothema 2009; 21:288-93.

57. González-Santa Cruz A. Propiedades psicométricas de escala de liderazgo destructivo y Cuestionario de Conductas Negativas-Revisado. Santiago: Universidad Diego Portales; 2019. 
58. Mikkelsen EG, Einarsen S. Bullying in Danish work-life: prevalence and health correlates. Eur J Work Organ Psychol 2001; 10:393-413.

59. Organización Mundial de la Salud. Depresión y otros trastornos mentales comunes. Estimaciones sanitarias mundiales. Geneva: Organización Mundial de la Salud; 2017.

60. Estancial C, de Azevedo R, Goldbaum M, Barros M. Psychotropic use patterns: are there differences between men and women? PLoS One 2018; 13:e0207921.

61. Drapeau A, Marchand A, Beaulieu-Prevost D. Epidemiology of psychological distress. In: L’Abate L, editor. Mental illnesses: understanding, prediction and control. London: InTechOpen; 2012. p. 105-34.

62. Einarsen S, Hauge LJ. Antecedents and consequences of workplace mobbing: a literature review. Rev Psicol Trab Organ 2006; 22:251-73.

63. Feijó F, Gräf D, Pearce N, Fassa A. Risk factors for workplace bullying: a systematic review. Int J Environ Res Public Health 2019; 16:1945.

64. Moreno B, Rodríguez A, Garrosa E, Morante ME. Antecedentes organizacionales del acoso psicológico en el trabajo: un estudio exploratorio. Psicothema 2005; 17:627-32.

65. Armstrong P, Messing K. Taking gender into account in occupational health research: continuing tensions. Policy and Practice in Health and Safety 2014; 12:3-16.

66. Baby M, Glue P, Carlyle D. "Violence is not part of our job": a thematic analysis of psychiatric mental health nurses' experiences of patient assaults from a New Zealand perspective. Issues Ment Health Nurs 2014; 35:647-55.
67. Comisión Económica para América Latina y el Caribe; Organización de las Naciones Unidas para la Alimentación y la Agricultura; ONU Mujeres; Programa de las Naciones Unidas para el Desarrollo; Organización Internacional del Trabajo. Trabajo decente e igualdad de género. Santiago: Comisión Económica para América Latina y el Caribe/Organización de las Naciones Unidas para la Alimentación y la Agricultura/ONU Mujeres/Programa de las Naciones Unidas para el Desarrollo/Organización Internacional del Trabajo; 2013.

68. Salin D. Workplace bullying and gender: an overview of empirical findings. In: D'Cruz P, Noronha E, Caponecchia C, Escartín J, Salin D, Tuckey MR, editors. Dignity and inclusion at work. Berlin: Springer; 2018. p. 1-31.

69. Notelaers G, Einarsen S. The world turns at 33 and 45: defining simple cutoff scores for the Negative Acts Questionnaire-Revised in a representative sample. Eur J Work Organ Psychol 2013; 22:670-82.

70. Geoffroy M, Chamberland L. Mental health implications of workplace discrimination against sexual and gender minorities: a literature review. Sante Ment Que 2015; 40:145-72.

71. Straiton ML, Aambø AK, Johansen R. Perceived discrimination, health and mental health among immigrants in Norway: the role of moderating factors. BMC Public Health 2019; 19:325. 


\section{Abstract}

Mobbing, or bullying in the workplace, has gained relevance in recent decades due to its growing magnitude and negative effects on workers' health. There are various approaches for studying the issue. However, thus far the evidence is scarce in Latin America and is focused on specific samples. This study aims to analyze workplace bullying and its association with mental health in the wage-earning population and to determine the extent to which this association is modified by gender. A survey was conducted with a sample of 1,995 male and female salaried workers in Chile's three main metropolitan areas (Greater Santiago, Greater Valparaiso, and Greater Concepcion) with three-stage random selection (blocks, households, and individuals). Prevalence rates for depressive symptoms, use of psychotropic medication, and stress were $10.9 \%, 12.8 \%$, and $13 \%$, respectively, and there was a strong relationship between mental health variables and workplace bullying, which persisted in the adjusted models. When comparing this association in the models stratified by gender, no significant differences were observed between men and women.

Workplace Violence; Mental Health; Occupational Health

\section{Resumo}

O assédio moral no trabalho ganhou relevância nas últimas décadas devido à sua crescente magnitude e às consequências negativas sobre a saúde dos trabalhadores, sendo que existem diversos enfoques para estudá-la. Entretanto, na América Latina, as evidências são raras e focalizadas em amostras específicas. O objetivo deste estudo é descrever o assédio no trabalho e a sua relação com a saúde mental nos funcionários, para determinar em que medida a relação se altera conforme o gênero. A pesquisa envolveu uma amostra de 1995 trabalhadores e trabalhadoras formais das três principais regiões metropolitanas do Chile (Grande Santiago, Grande Valparaíso e Grande Concepción) selecionados ao acaso, em três etapas (quarteirão, unidades habitacionais e indivíduos). A prevalência de sintomas depressivos, consumo de psicotrópicos e distresse foi de 10,9\%, 12,8\% e 13\% respectivamente, na amostra total, e existe uma forte relação entre variáveis de saúde mental e assédio moral no trabalho, ainda nos modelos ajustados. A comparação desta relação nos modelos estratificados por gênero não mostrou diferenças significativas entre homens e mulheres.

Violência no Trabalho; Saúde Mental; Saúde do Trabalhador
Recibido el 05/Mar/2020

Versión final presentada el 30/Jun/2020

Aprobado el 17/Jul/2020 